\title{
Population pharmacokinetics of methylphenidate hydrochloride extended-release multiple-layer beads in pediatric subjects with attention deficit hyperactivity disorder
}

\author{
This article was published in the following Dove Press journal: \\ Drug Design, Development and Therapy \\ 26 May 2015 \\ Number of times this article has been viewed
}

\author{
Nathan S Teuscher' \\ Akwete Adjei $^{2}$ \\ Robert L Findling ${ }^{3,4}$ \\ Laurence L Greenhill ${ }^{5}$ \\ Robert J Kupper ${ }^{2}$ \\ Sharon Wigal ${ }^{6}$ \\ 'PK/PD Associates, Trophy Club, \\ TX, ${ }^{2}$ Rhodes Pharmaceuticals L.P., \\ Coventry, RI, ${ }^{3}$ Department of \\ Psychiatric Services and Research, \\ Kennedy Krieger Institute, Baltimore, \\ MD, ${ }^{4}$ Department of Psychiatry \\ and Behavioral Sciences, Johns \\ Hopkins University, Baltimore, MD, \\ ${ }^{5}$ Department of Psychiatry, Division \\ of Child and Adolescent Psychiatry, \\ New York State Psychiatric Institute, \\ Columbia University, New York, NY, \\ ${ }^{6}$ AVIDA Inc., Newport Beach, CA, \\ USA
}

\begin{abstract}
A new multilayer-bead formulation of extended-release methylphenidate hydrochloride (MPH-MLR) has been evaluated in pharmacokinetic studies in healthy adults and in Phase III efficacy/safety studies in children and adolescents with attention deficit hyperactivity disorder (ADHD). Using available data in healthy adults, a two-input, one-compartment, first-order elimination population pharmacokinetic model was developed using nonlinear mixed-effect modeling. The model was then extended to pediatric subjects, and was found to adequately describe plasma concentration-time data for this population. A pharmacokinetic/ pharmacodynamic model was also developed using change from baseline in the ADHD Rating Scale (ADHD-RS)-IV total scores from a pediatric Phase III trial and simulated plasma concentration-time data. During simulations for each MPH-MLR dose level (10-80 mg), increased body weight resulted in decreased maximum concentration. Additionally, as maximum concentration increased, ADHD-RS-IV total score improved (decreased). Knowledge of the relationship between dose, body weight, and clinical response following the administration of MPH-MLR in children and adolescents may be useful for clinicians selecting initial dosing of MPH-MLR. Additional study is needed to confirm these results.
\end{abstract}

Keywords: population pharmacokinetics, Aptensio XR ${ }^{\mathrm{TM}}$, MPH-MLR, methylphenidate

\section{Introduction}

A novel multilayer-release (MLR) bead formulation of methylphenidate (MPH) hydrochloride contained in a hard gelatin capsule (MPH-MLR; Aptensio XR ${ }^{\mathrm{TM}}$ ) is in late-stage clinical development by Rhodes Pharmaceuticals L.P. (Coventry, RI, USA) for the treatment of attention deficit hyperactivity disorder (ADHD) in children (6-12 years of age), adolescents (13-17 years of age), and adults ( $\geq 18$ years of age). ${ }^{1} \mathrm{MPH}-$ MLR was designed as an alternative to multiple-dose immediate-release (IR) MPH by providing a biphasic plasma concentration-time profile when given as a single daily dose. The MLR bead system used in MPH-MLR provides a rapid initial release of $37 \%$ of the total MPH dose, delivering a peak concentration comparable with that of IR MPH, as was observed in four Phase I trials of children with ADHD and healthy young adults. ${ }^{2-5}$ In three Phase II studies of ADHD in children, adolescents, ${ }^{6,7}$ and adults ${ }^{8}$ this formulation of once-daily MPH-MLR provided a sustained duration of effect with significant improvements in behavior and cognitive measures and a sideeffect profile that was consistent with the known adverse-event profile of MPH.

Maximum plasma drug concentration $\left(\mathrm{C}_{\max }\right)$ and area under the plasma drug concentration-time curve (AUC) are not the only relevant pharmacokinetic (PK) parameters
Correspondence: Akwete Adjei Rhodes Pharmaceuticals L.P., 498 Washington Street, Coventry, RI 028I6, USA

$\mathrm{Tel}+\mathrm{I} 4012629408$

Fax + I 40I 262 940I

Email akwete.adjei@pharma.com 
for estimation of clinical response profile or discrimination between MPH extended-release (ER) formulations. They do not describe the onset and duration of the therapeutic effect from MPH ER formulations, nor do they take into account the shape of the concentration-time curve., ${ }^{9,10}$ The US Food and Drug Administration recommends use of partial AUC (pAUC) metrics in addition to $\mathrm{C}_{\max }$ and AUC for these purposes..$^{9,11}$ Furthermore, the temporal clinical response profile of $\mathrm{MPH}$ in patients with ADHD can be related to pAUC by use of a

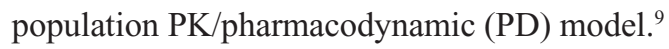

Several MPH PK/PD models have been described. Shader et $\mathrm{a}^{12}$ developed a population $\mathrm{PK}$ model using a single plasma MPH concentration from 273 children and adolescents who were designated as "good responders" to IR MPH treatment to characterize PK parameters for the population. A good correlation $(r=0.83$ ) was found between the model prediction and measured plasma MPH concentrations from 16 participants in a substudy who returned 4 weeks later for an additional blood sample. Another PK model was used to compare MPH PK in preschoolers $(n=14)$ and children 6-8 years of age $(\mathrm{n}=8) \cdot{ }^{13,14}$ In this model, MPH AUC was related to age; given the same weight-based dose, preschool children had greater MPH exposure than school-age children, the difference of which was not explained simply by weight-based differences. PK data from a meta-analysis of published Phase I studies of MPH ER formulations in healthy adult volunteers were used to develop a PD model described by Kimko et al. ${ }^{15}$ In addition, the model incorporated clinical data (Swanson, Kotkin, Agler, M-Flynn, and Pelham [SKAMP] rating scale scores and Permanent Product Measure of Performance scores) from two pediatric studies with the same study design. The model was able to illustrate the relationships between the mean concentration-time profiles in adults and the time course of clinical response in pediatric subjects.

While previous reports have described the systemic PK of MPH, this is the first report of a population PK and PK/ PD model for this novel MPH-MLR product in late-stage development. Several objectives were established for this analysis. The first was to develop an MPH-population PK model using data from healthy adult volunteers to simulate concentration-time data for several MPH-MLR dosing levels $(10,15,20,30,40$, and $60 \mathrm{mg})$. The second objective was to develop a pediatric population PK model based on the adult model. The predicted concentration-time data from adults was used to develop a PK/PD model for pediatric study participants that described the change from baseline in the ADHD Rating Scale (ADHD-RS)-IV total score. Finally, the $\mathrm{PK} / \mathrm{PD}$ model was used to simulate the change from baseline in ADHD-RS-IV total score for pediatric subjects (6-18 years of age, inclusive) for all strengths of MPH-MLR across a range of body weights (70-150 lb [32-68 kg]).

\section{Materials and methods}

Population PK models are used to supplement data collected during studies for clinical trials. ${ }^{16}$ For this analysis, adult and pediatric population PK models and a PK/PD model were developed. Data from four studies were used to construct the models. This was a secondary analysis of clinical trials; detailed institutional review board approval and informed consent were not applicable.

\section{Data sources}

The adult PK model included the concentration-time data from two PK studies in healthy adult volunteers. ${ }^{4,5}$ The first study was a single-center, randomized, open-label, single-dose, three-period crossover study that assessed the relative bioavailability of three doses (4 hours apart) of IR methylphenidate (Ritalin; Novartis Pharmaceuticals, East Hanover, NJ, USA) $25 \mathrm{mg}$ and a single dose of MPH-MLR $80 \mathrm{mg}$, each given in the fasted state (single-dose study). ${ }^{5}$ Blood samples were obtained before dosing ( $\leq 15$ minutes) and at $0.5,1,1.5,2,2.5,3,4,4.5,5,5.5,6,6.5,7,8,8.5,9$, $9.5,10,10.5,12,15,19$, and 24 hours postdose. The second study was a single-center, randomized, open-label, single- and multiple-dose, two-period crossover study that assessed the relative bioavailability of three doses (4 hours apart) of IR methylphenidate $25 \mathrm{mg}$ daily and a single daily dose of MPHMLR $80 \mathrm{mg}$ under fed conditions (multidose study). ${ }^{4}$ The first assessment was done following a high-fat breakfast on day 1 , with a second assessment on day 4 in the fed state after a standard meal. Each assessment day required obtainment of serial blood samples just before dosing ( $\leq 15$ minutes) and at $0.5,1,1.5,2,2.5,3,4,4.5,5,5.5,6,6.5,7,8,8.5,9,9.5,10$, $10.5,12,15,19$, and 24 hours postdose. In addition, blood samples were obtained at 4, 8, 12, 16, and 24 hours postdose on days 2 and 3. Plasma from blood samples obtained in both studies was extracted and analyzed to determine MPH concentration using a fully validated liquid chromatography analysis method (calibration range $0.05-25 \mathrm{ng} / \mathrm{mL}$ ). ${ }^{2}$

The pediatric data came from two studies. MPH plasma concentration-time data included in the model were obtained from a single-center, randomized, open-label, two-way crossover study that compared the PKs of MPH-MLR (10, 15, 20, 30 , and $40 \mathrm{mg}$ strengths) and IR methylphenidate (10 and $20 \mathrm{mg}$ strengths) in children 6-12 years of age with ADHD. ${ }^{2}$ In each of the two dosing phases, serial plasma samples were 
obtained on the day of dosing at time 0 (predose) and at 1 , $2,3,4,5,6,8,10,12$, and 24 hours postdose. Plasma MPH concentrations were determined by use of a high-performance liquid chromatographic method with MPH detection by tandem mass spectrometry using MPH-d3 as the internal standard (as previously described). ${ }^{2}$ The second study, a parallel, randomized, double-blind, multicenter, placebo-controlled, forced-dose, Phase III study, which evaluated the safety and efficacy of MPH-MLR in children and adolescents 6-18 years of age (ClinicalTrials.gov identifier NCT01239030), supplied the ADHD-RS-IV total score data. ADHD-RS-IV total scores were obtained at baseline, end of week 1 (end of double-blind phase), and weeks 2, 3, 4, 8, and 12 (open-label phase).

\section{Population PK modeling}

The adult pharmacostatistical model used in this study has been briefly described. ${ }^{5}$ Both the adult and pediatric population PK models were built using nonlinear mixed-effect modeling with first-order conditional maximum-likelihood estimation using Phoenix NLME 1.3 (Pharsight Corporation, St Louis, MO, USA). MPH plasma concentrations following the administration of MPH-MLR were described by two parallel inputs into a central compartment and linear elimination from the central compartment (Figure 1). Absorption of MPH-MLR was best described using a first-order rate constant, although a zero-order rate constant was also explored. Model suitability was explored using a posterior predictive check method, where a majority of the observed data fell within the fifth and 95th percentiles of the predicted concentrations.

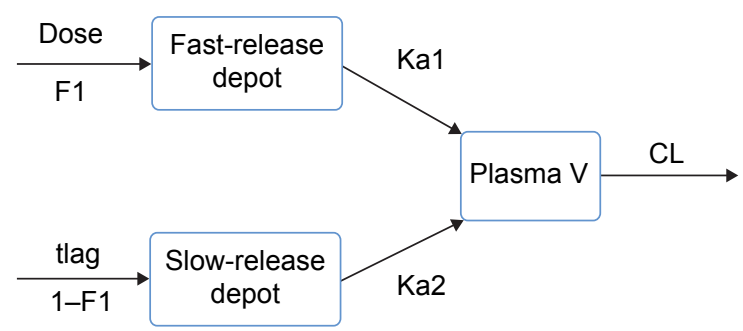

Figure I Structural PK model for MPH-MLR that includes IR and ER components of $\mathrm{MPH}$.

Notes: The fast-release depot represents the IR overcoating that was readily absorbed into the plasma using a first-order rate constant (Kal). The fraction of $\mathrm{MPH}$ dose released from the IR coating was estimated with the relative bioavailability parameter $(\mathrm{FI})$. The slow-release depot represents the ER core that slowly released the drug following water permeation into the product core. The slow release also followed first-order absorption kinetics (Ka2). The time required for water to permeate the product core was represented with a lag time (tlag). The fraction of MPH dose released from the ER core was estimated as I-FI. First-order elimination from the central compartment adequately described the elimination kinetics of MPH from the plasma. This elimination process was described with a volume of distribution $(\mathrm{V})$ and clearance $(\mathrm{CL})$.

Abbreviations: ER, extended release; IR, immediate release; MPH-MLR, methylphenidate extended-release multilayer beads; PK, pharmacokinetic; V, volume of distribution.
Monte Carlo simulations were performed with the final model using the final PK parameter estimates, between subject variability, and residual variability. Eight simulations were performed for a single dose of MPH-MLR at the 10, $15,20,30,40,50,60$, and $80 \mathrm{mg}$ dosage strengths. Simulations were performed with the same number of subjects as the single-dose PK study in replicates of 100 for each dosage strength.

All parameters were assumed to be log-normally distributed and had exponential between-individual variability terms (Equation 1):

$$
P_{\mathrm{i}}=P * e^{\eta^{p}}
$$

where:

$P_{\mathrm{i}}=$ true parameter value for individual $\mathrm{i}$,

$P=$ typical value (population mean) of the parameter, and

$\eta_{\mathrm{i}}^{p}=$ difference between the true value for individual $\mathrm{i}$ and the typical value for the population, with a mean of 0 and a variance of $\omega^{2}$.

For the purpose of this analysis, additive (Equation 2) and proportional (Equation 3) residual error models were evaluated:

$$
\begin{gathered}
C_{\mathrm{i} j}=\hat{C}_{\mathrm{i} j}+\varepsilon_{1 \mathrm{ij}} \\
C_{\mathrm{i} j}=\hat{C}_{\mathrm{i} j} *\left(1+\varepsilon_{2 \mathrm{ij}}\right)
\end{gathered}
$$

where:

$C_{\mathrm{i} j}=j$ th measured concentration for individual $\mathrm{i}$,

$\hat{C}_{\mathrm{i} j}=j$ th model predicted concentration for individual $\mathrm{i}$,

$\varepsilon_{1 \mathrm{ij}}=$ additive residual error for the $j$ th concentration for individual $\mathrm{i}$, and is normally distributed with a mean of 0 and a variance of $\omega_{2}^{2}$, and

$\varepsilon_{2 \mathrm{ij}}=$ proportional residual error for the $j$ th measurement for individual $\mathrm{i}$, and is normally distributed with a mean of 0 and a variance of $\omega_{1}{ }^{2}$.

Hypothesis testing was performed using the likelihoodratio test to discriminate among alternative hierarchical models. When comparing alternative models, the difference in the objective function was approximately $\chi^{2}$ distributed with $\mathrm{n}$ degrees of freedom, where $\mathrm{n}$ is the difference in the number of parameters between the hierarchical models. A decrease of 6.68 in the value of the objective function value (which is minus twice the maximum logarithm of the likelihood of the data) was significant under the likelihood-ratio test $(\mathrm{n}=1, P<0.01)$. In modeling the data, it was assumed that: 1) sampling times 
and dosing histories were recorded accurately, and any error associated with these values did not lead to significant modeling bias; and 2) the proportion of drug in the IR layer $\left(\mathrm{F}_{1}\right)$ was consistent across all dosage strengths.

The model was evaluated by inspection of the following diagnostic plots for goodness of fit: population typical predicted versus observed dependent variables; individual predicted versus observed dependent variables; individual weighted residuals as a function of time, population typical predicted dependent variable, single and multiple dosing, and daily dose; and individual predicted and observed plasma concentrations as a function of time.

The pediatric population PK model was based on the adult model. Potential covariates were examined using forward addition $(P<0.01)$ and backward deletion $(P<0.001)$ in a stepwise manner using the likelihood-ratio test to compare hierarchical models. The following covariates were examined for potential effects on volume and clearance: body weight, height, and body mass index. The final model included body weight as a covariate of MPH clearance, as shown in Equation 4:

$$
C L_{\mathrm{i}}=C L_{T V} * W T^{\theta} e_{\mathrm{i}}^{\eta_{\mathrm{i}}}
$$

where:

$C L_{\mathrm{i}}=$ true clearance for individual $\mathrm{i}$,

$C L_{T V}=$ typical value (population mean) for clearance,

$W T=$ body weight for individual $\mathrm{i}$,

$\theta=$ effect of body weight on clearance, and

$\eta_{i}^{C L}=$ difference between the true value for individual $i$ and the typical value for the population, with a mean of 0 and a variance of $\omega^{2}$.

\section{PK/PD modeling}

The pediatric population PK/PD model was developed using change from baseline ADHD-RS-IV total score from a Phase III trial as a response variable and simulated $\mathrm{C}_{\max }$ values for subjects from the pediatric population PK model. Plasma MPH concentration-time data for each treatment week were simulated using the developed pediatric population PK model, and peak exposure $\left(\mathrm{C}_{\max }\right)$ and total exposure (AUC) were estimated from the predicted data. A population $\mathrm{PK} / \mathrm{PD}$ model was then developed (Equation 5) using $\mathrm{C}_{\max }$ as the independent variable $(C)$ and change from baseline ADHD-RS-IV total score as the dependent variable $(E)$ :

$$
E=\frac{E_{\max } * C}{E C_{50}+C}
$$

where:

$E_{\max }=$ maximum change from baseline in ADHD-RS-IV total score, and

$E C_{50}=\mathrm{C}_{\max }$ value that provides $50 \%$ of the maximum change from baseline in ADHD-RS-IV total score.

The PK/PD model included between-individual variability parameters for $E_{\max }$ and $E C_{50}$, and a proportional residual error parameter. The impact of body weight-based and fixed dosing of MPH-MLR was evaluated using a Monte Carlo simulation approach, performed with the final population PK/PD model for MPH-MLR using PK and PD parameters, between-individual variability, and residual variability. Combinations of simulations were run-in replicates of 200 for subjects with body weights ranging from 70 to $150 \mathrm{lb}$ and MPH-MLR dose strengths of 10, 15, 20, 30, 40, 50,60 , and $80 \mathrm{mg}$.

\section{Results}

The final-analysis data set for the adult population PK model included only data from subjects receiving MPH-MLR. A total of 1,124 MPH concentration measurements from 25 subjects in the single-dose study were used. Following the development of the adult population PK model, simulated profiles from the PK model were compared with actual concentration measurements from the multiple-dose study to validate the capability of the PK model in predicting multiple-dose profiles accurately.

The final-analysis data set for the pediatric population PK model included $154 \mathrm{MPH}$ concentration measurements from 17 subjects, and the pediatric population PK/PD analysis included 1,767 change-from-baseline ADHD-RS-IV total scores from 269 study participants.

\section{Population PK modeling}

For the adult base model, the low coefficient of variation $(<12 \%)$ for all structural parameters indicated that precision was high, ${ }^{5}$ and diagnostic plots showed a good fit of the model to the MPH concentrations. A posterior predictive check of the adult population PK model comparing the MPH-MLR $80 \mathrm{mg}$ simulation to observed data from the single-dose PK study found most of the data to be within the fifth and 95th percentiles of the simulated data, suggesting that the model was adequate for simulations. Additionally, the adult-based model was used to simulate multiple doses and compared with observed data from a multiple-dose study. Most observed data were within the fifth and 95th percentiles of the simulated data, suggesting that the model was useful for multiple-dose simulations. 
The same two-input, one-compartment, first-order elimination model used for adult subjects adequately described the plasma concentration-time data following the administration of a single dose of MPH-MLR to pediatric subjects. The final population PK model was parameterized using: the fraction drug dose in the IR component of MPH-MLR (F1); the absorption-rate constant from the IR (Ka1) and ER (Ka2) components of MPH-MLR, lag time, clearance (CL), and apparent volume of distribution (V); and between-individual variability parameters for Ka1, Ka2, CL, and V. Parameter estimates from the additive and proportional residual error models were similar; the proportional residual error model was selected for evaluation of covariates because of a slightly lower log-likelihood value and equivalent parameter estimates. The diagnostic plots showed a good fit of the final pediatric population PK model to MPH concentrations. Body weight was found to be a significant covariate of MPH clearance in pediatric subjects.

Final parameter estimates for the pediatric PK model are shown in Table 1. Figure 2 shows the goodness-of-fit relationship between the individual population PK model observations versus time after dose. Residuals were uniformly distributed with time and MPH concentrations with no obvious bias. A posterior predictive check of the final pediatric PK model found that the fifth and 95th quartiles of the observed data were contained within the $90 \%$ confidence intervals for the fifth and 95 th prediction intervals, suggesting

Table I Final pediatric population PK-model parameter estimates

\begin{tabular}{ll}
\hline $\begin{array}{l}\text { Final pediatric population } \\
\text { PK model }\end{array}$ & $\begin{array}{l}\text { Proportional residual } \\
\text { error }\end{array}$ \\
Log likelihood & $-34 \mathrm{I} .4342$ \\
Structural model parameters & Estimate $(\% \mathrm{CV})$ \\
$\mathrm{CL}(\mathrm{L} / \mathrm{h})$ & $\mathrm{I} .3(68.8)$ \\
$\mathrm{V}(\mathrm{L})$ & $64.7(25.8)$ \\
$\mathrm{FI}$ & $0.65(10.5)$ \\
$\mathrm{KaI}(\mathrm{I} / \mathrm{h})$ & $0.25(26.6)$ \\
$\mathrm{Ka} 2(\mathrm{I} / \mathrm{h})$ & $0.16(3 \mathrm{I} .8)$ \\
tlag $(\mathrm{h})$ & $5.75(3.6)$ \\
$\theta(\mathrm{effect}$ of body weight on CL) & $1.53(\mathrm{I} 2.9)$ \\
Between-individual variability & Estimate (\% shrinkage)* \\
parameters & \\
$\omega \mathrm{CL}$ & $0.057(\mathrm{I})$ \\
$\omega \mathrm{V}$ & $0.096(29)$ \\
Residual variability parameter & Estimate $(\% \mathrm{CV})$ \\
$\varepsilon$ & $\mathrm{I} .94(6.2)$ \\
\hline
\end{tabular}

Note: *Shrinkage calculated as I- standard deviation/estimate.

Abbreviations: $\mathrm{CV}$, coefficient of variation (calculated as standard deviation/estimate; $\mathrm{CL}$, clearance; FI, IR layer; IR, immediate release; Kal, absorption-rate constant from immediate-release component; Ka2, absorption-rate constant from extendedrelease component; PK, pharmacokinetic; tlag, lag time; $\mathrm{V}$, volume of distribution.

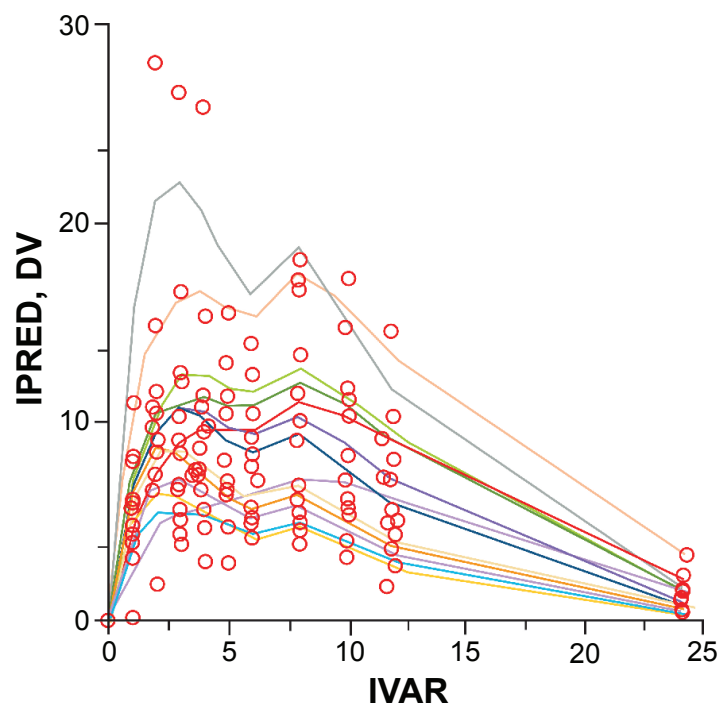

Figure 2 Final pediatric population pharmacokinetic model observations and individual predictions versus time after dose.

Notes: Model observations are actual concentration-time data and are displayed with open circles. Individual predicted concentration-time profiles are displayed with lines (colored by subject).

Abbreviations: DV, observations; IPRED, independent predicted concentrations; IVAR, independent variable.

that the model is adequate for simulating MPH concentrations in pediatric patients.

\section{PK/PD modeling}

Change from baseline ADHD-RS-IV total score response data were fit to an $E_{\max }$ model that used predicted $\mathrm{C}_{\max }$ as the independent variable using a first-order conditional estimation with interaction method. The final parameters for the PK/PD model are shown in Table 2. The maximum change from baseline ADHD-RS-IV total score was -34.96 , and the $\mathrm{C}_{\max }$ required to elicit $50 \%$ of the maximal effect was $5.77 \mathrm{ng} / \mathrm{mL}$. The diagnostic plot showed a good fit of the pediatric population PK/PD model to the change from baseline ADHD-RS-IV total score (Figure 3). Residuals were uniformly distributed with time and change from baseline ADHD-RS-IV total score with no obvious bias.

Due to the relationship between body weight and MPH clearance, there is an inverse correlation between body weight and maximum MPH exposure, such that as body weight increases at a given dose strength, the $\mathrm{C}_{\text {max }}$ decreases. Additionally, $\mathrm{C}_{\max }$ was negatively correlated with the change from baseline ADHD-RS-IV total score: as $\mathrm{C}_{\max }$ increased, the ADHD-RS-IV total score decreased (indicating improvement). These relationships are shown by plotting the mean change from baseline ADHD-RS-IV total score versus body weight using a single-dose strength (Figure 4A) or the mean change from baseline ADHD-RS-IV total score versus 
Table 2 Pediatric population PK/PD-model parameter estimates

\begin{tabular}{ll}
\hline Pediatric population & Proportional residual \\
PK/PD model & error \\
Log likelihood & $-3,633.13$ \\
Structural model parameters & Estimate $(\% \mathrm{CV})$ \\
$E_{\max }$ & $-34.96(6.8)$ \\
$E C_{50}(\mathrm{ng} / \mathrm{mL})$ & $5.77(\mathrm{I} 2.6)$ \\
Between-individual variability & Estimate (\% shrinkage)* \\
parameters & \\
$\omega E_{\max }$ & $0.028(76)$ \\
$\omega E C_{50}$ & $1.01(39)$ \\
Residual variability parameter & Estimate $(\% \mathrm{CV})$ \\
$\varepsilon$ & $0.44(4.9)$ \\
\hline
\end{tabular}

Note: *Shrinkage calculated as I- standard deviation/estimate.

Abbreviations: CV, coefficient of variation (calculated as standard deviation/ estimate; $E_{\max }$, maximum change from baseline; $E C_{50}$, maximum concentration value that provides $50 \%$ of the maximum change from baseline; PD, pharmacodynamic; PK, pharmacokinetic.

dose strength using a single body weight (Figure 4B). The simulated response profiles for body weight ranging from 70 to $150 \mathrm{lb}$ across all MPH-MLR dose strengths showed that, in general, response increased with increasing dose strength and decreasing body weight (Figure 5). Clinical management of symptoms requires a significant decrease in ADHD-RS-IV total score, although the magnitude of change required for clinical improvement may vary depending on the severity of the initially presenting symptoms. In the pediatric studies, the mean (standard deviation) ADHD-RS-IV total score

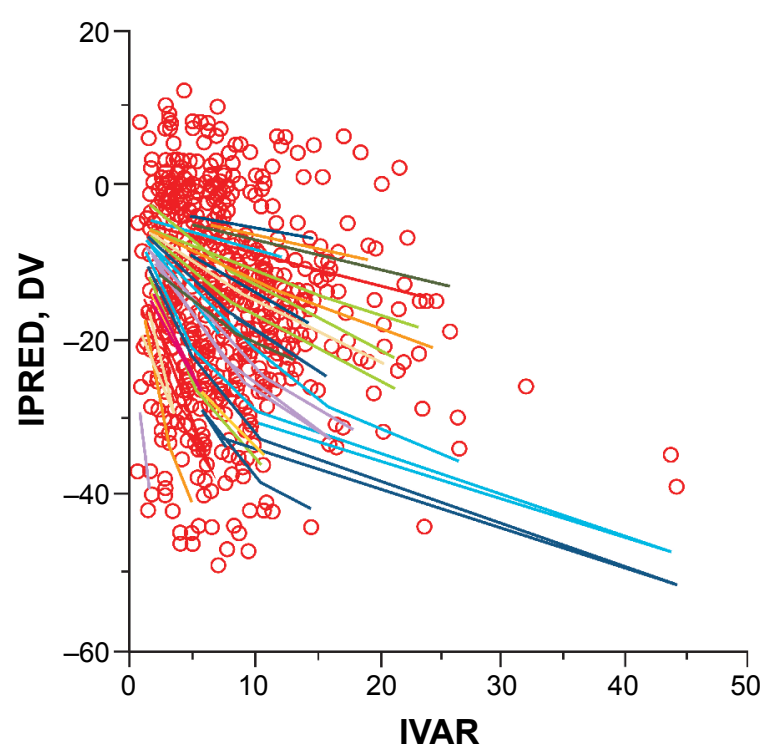

Figure 3 Individual pediatric population PK/PD model observations versus the independent variable (simulated $\mathrm{C}_{\max }$ ).

Notes: Model observations are actual PK/PD data and are displayed with open circles. Individual predicted PK/PD profiles are displayed with lines (colored by subject).

Abbreviations: $C_{\max }$, maximum concentration; DV, observations; PD, pharmacodynamic; PK, pharmacokinetic; IPRED, independent predicted responses; IVAR, independent variable.
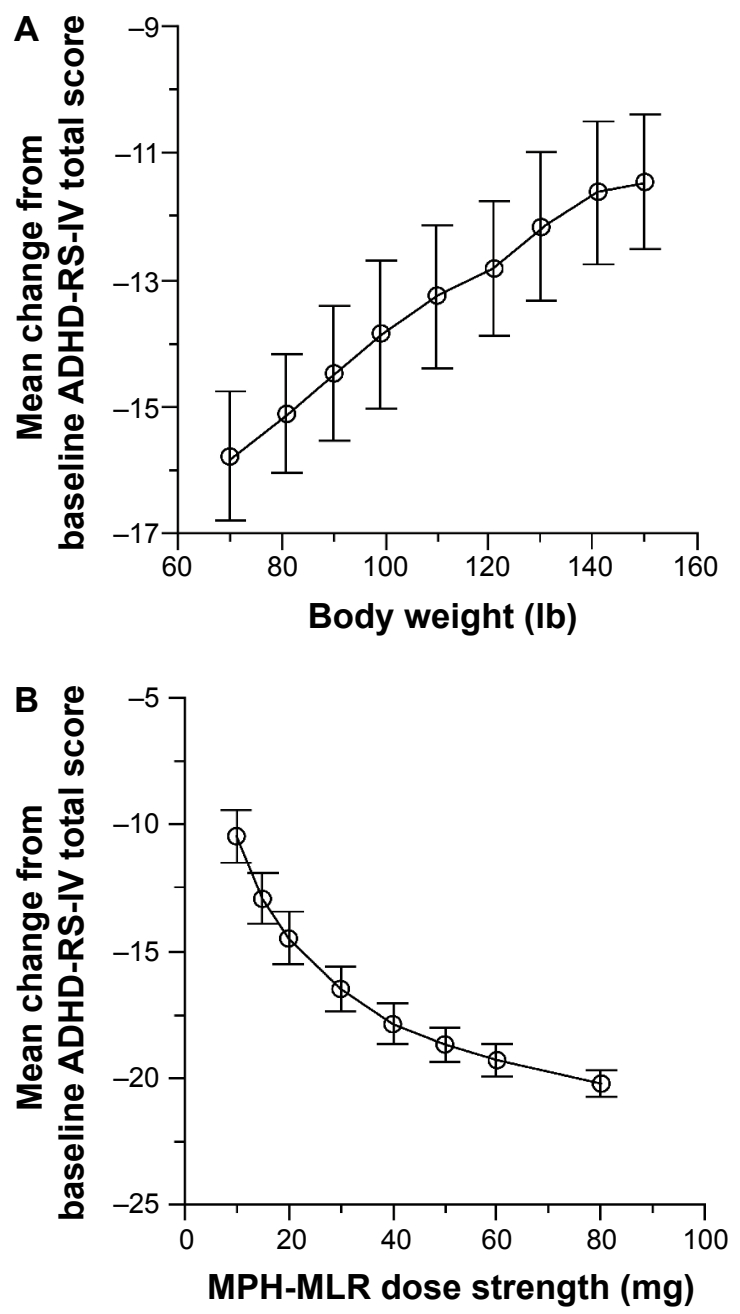

Figure 4 Simulated mean ( \pm standard deviation) change from baseline ADHD-RS-IV total score.

Note: (A) Response from MPH-MLR $20 \mathrm{mg}$ across a range of body weights; (B) response for a $90 \mathrm{lb}$ patient across a range of MPH-MLR dosage strengths. Abbreviations: ADHD-RS-IV, Attention Deficit Hyperactivity Disorder Rating Scale-IV; MPH-MLR, methylphenidate extended-release multilayer beads.

at baseline was 36.3 (9.32), and an 18-point reduction in ADHD-RS-IV total score represented a 50\% reduction from the mean baseline score. This 50\% reduction in ADHDRS-IV total score was considered clinically relevant for the purposes of this simulation. Using the 18-point reduction in ADHD-RS-IV total score as a threshold for a beneficial response, simulations of the MPH-MLR dose strength required to elicit such an effect for a range of body weights is shown in Table 3.

\section{Discussion}

A two-input, one-compartment, first-order elimination population PK model adequately described MPH concentrations following the administration of MPH-MLR in healthy adults. The same model sufficiently described the plasma 


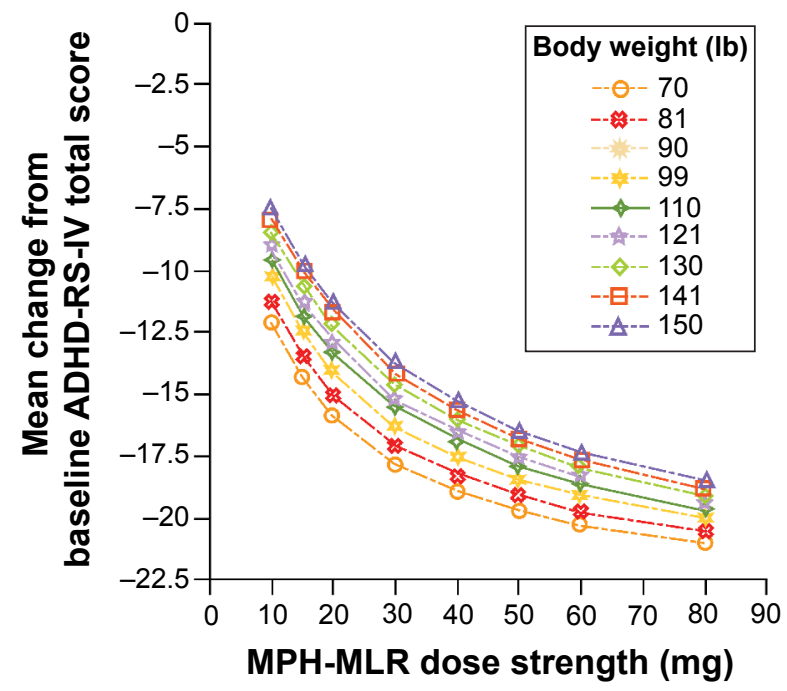

Figure 5 Simulated mean change from baseline ADHD-RS-IV total score for a range of body weights (70-150 lb) and MPH-MLR dose strengths (10-80 mg). Abbreviations: ADHD-RS-IV, Attention Deficit Hyperactivity Disorder Rating Scale-IV; MPH-MLR, methylphenidate extended-release multilayer beads.

concentration-time data following the administration of a single dose of MPH-MLR to pediatric subjects. MPH-MLR $60 \mathrm{mg}$ ( $\sim 22 \mathrm{mg}$ as the IR component) yielded early MPH exposure equivalent to IR MPH $25 \mathrm{mg}$ when $\mathrm{pAUC}_{0-4}$ results were compared. ${ }^{5}$ The differences in concentrationtime profiles between IR MPH and MPH-MLR have been discussed previously. ${ }^{4,5}$ In both PK models, a relationship between body weight and clearance was noted, suggesting that heavier individuals have greater clearance, ultimately resulting in lower systemic exposure to $\mathrm{MPH}$. The increase in clearance resulted in a lower $\mathrm{C}_{\max }$ and smaller AUC, indicating that two individuals who receive the same MPH-MLR dose strength will experience different peak MPH exposures, with the heavier person having lower MPH concentrations.

Table 3 Simulations of MPH-MLR dose required to achieve an I8-point reduction in ADHD-RS-IV total score at selected body weights

\begin{tabular}{lll}
\hline $\begin{array}{l}\text { Body } \\
\text { weight, } \mathbf{~ k g}\end{array}$ & $\begin{array}{l}\text { MPH-MLR } \\
\text { dose }(\mathbf{m g})\end{array}$ & $\begin{array}{l}\text { Mean change from baseline } \\
\text { ADHD-RS-IV total score }\end{array}$ \\
\hline $32(70 \mathrm{lb})$ & 40 & $-18.8 \mathrm{I}$ \\
$36(80 \mathrm{lb})$ & 40 & -18.28 \\
$4 \mathrm{I}(90 \mathrm{lb})$ & 50 & -18.67 \\
$45(100 \mathrm{lb})$ & 50 & -18.35 \\
$50(110 \mathrm{lb})$ & 60 & -18.52 \\
$54(120 \mathrm{lb})$ & 60 & -18.35 \\
$59(130 \mathrm{lb})$ & 80 & -19.02 \\
$64(140 \mathrm{lb})$ & 80 & -18.67 \\
$68(150 \mathrm{lb})$ & 80 & -18.46 \\
\hline
\end{tabular}

Abbreviations: ADHD-RS-IV, Attention Deficit Hyperactivity Disorder Rating Scale-IV; MPH-MLR, methylphenidate extended-release multilayer beads.
Intuitively, this finding indicates that heavier pediatric patients might require larger doses than patients with lighter body weights to achieve similar systemic exposure.

The relationship between body weight and clinical response for MPH has not been consistently supported in the literature. Likewise, no relationship between body weight and clinical response was noted in a study of MPH in 76 children with ADHD. ${ }^{17}$ In other studies, MPH clearance correlated with age: lower drug clearance was noted in younger patients, resulting in higher drug exposure. ${ }^{18}$ In general, age and body weight are collinear in older pediatric patients. While PK data are available for many $\mathrm{MPH}$ formulations and may link exposure and body weight, data have not been linked to clinical response.

Our population $\mathrm{PK} / \mathrm{PD}$ analysis was designed to inform on the expected efficacy response to MPH-MLR across a range of body weights and dose strengths. Additionally, the simulations permitted exploration of the potential effects of MPH-MLR under circumstances that were not directly studied in clinical trials. Results of the pediatric population PK/PD model showed that peak MPH concentrations drive clinical efficacy measured by ADHD-RS-IV total score. The combined PK/PD model simulations provide evidence to suggest that weight may have a significant impact on the efficacy of MPH-MLR and should be taken into consideration with initial dosing.

The model suggests that at the same dose strength, children with higher body weight will be more likely to experience reduced symptom control relative to children with lower body weight, and increasing the dose will generally lead to more symptom reductions, as evidenced by a decrease in ADHD-RS-IV total score. However, since this assumption is based on simulated scenarios, further research will need to prospectively test this finding.

In the PK/PD analysis reported here, ADHD-RS-IV total scores were used as the efficacy parameter. The ADHDRS-IV total score used in this paper is a measure of improvement in overall ADHD symptoms over a treatment period compared with baseline values (ie, several weeks, which may include dose optimization at some point). ${ }^{19}$ Similar results were obtained using SKAMP scores (data not presented). SKAMP scores are specific to the analog classroom study setting, in which subjects are assessed for efficacy based on attention and deportment independently with or without drug treatment. ${ }^{20}$ Therefore, the ADHD-RS-IV total score could be considered more representative of overall ADHD symptoms in patients. Improvements in ADHD-RS-IV total score represent improvements in the patient's symptoms 
that may be manifest both within and outside the analog classroom setting.

The model simulation predicts that a clinically meaningful decrease in ADHD-RS-IV total score of 18 points can be achieved with MPH-MLR doses of $40 \mathrm{mg}$ in subjects that weigh at least $80 \mathrm{lb}$. Heavier subjects weighing $150 \mathrm{lb}$ or more appeared to require the $80 \mathrm{mg}$ strength of MPH-MLR to achieve the same 18-point reduction in ADHD-RS-IV total score. The availability of MPH-MLR dosage strengths of $10-80 \mathrm{mg}$ will provide the clinician with the ability to conveniently treat patients across a wide range of body weights.

The PK and PD data used in this analysis were collected in separate studies, which is a limitation. Predicted concentrations instead of actual concentrations were used in the PK/PD model. While every effort was made to ensure that the predictive power of the final pediatric PK model was adequate, differences may exist between the predicted concentrations and actual concentrations in the studies where efficacy was measured. This modeling was constructed to evaluate data for children and adolescents, and should not be extended to adults in the absence of additional studies. Additionally, these analyses focus on symptom reduction and do not include data regarding tolerability or adverse events. Although patient care requires individual assessments of response, mean response information can be a valuable guide for physicians as they customize therapy to individual patients. Future studies that can include both PK and efficacy measures would be useful in further exploring the relationships suggested by these models. These results will need to be confirmed in prospective studies.

In conclusion, the MPH-population PK model developed for adults was successfully extended to children and adolescents. Additionally, an $E_{\max }$ PK/PD model was developed to describe the change from baseline ADHD-RS-IV total score when $\mathrm{MPH} \mathrm{C}_{\max }$ was used as the independent variable. Based on the simulations, it appears that children weighing 150 $\mathrm{lb}$ or more may require escalation to the $80 \mathrm{mg}$ MPH-MLR dosage to achieve meaningful amelioration of ADHD symptomatology. Confirmation of these results using prospective clinical data would be useful to clinicians and patients.

\section{Acknowledgments}

The authors appreciate the contribution of pharmacokinetic data collected by Declan Quinn that were included in the model. Medical writing support was provided by Linda Wagner and Malcolm Darkes, medical writers at Excel Scientific Solutions, and funded by Rhodes Pharmaceuticals L.P.

\section{Disclosure}

The studies used for this model, as well as this analysis, were funded by Rhodes Pharmaceuticals L.P. NST is a consultant for Rhodes Pharmaceuticals L.P. AA is the executive director of product development at Rhodes Pharmaceuticals L.P. RLF receives or has received research support, acted as a consultant, and/or served on a speakers bureau for Alcobra, American Academy of Child and Adolescent Psychiatry, American Physician Institute, American Psychiatric Press, Bracket, Bristol-Myers Squibb, Clinsys, CogCubed, Cognition Group, Coronado Biosciences, Dana Foundation, Elsevier, Forest, Guilford Press, Johns Hopkins University Press, KemPharm, Lundbeck, Merck, National Institutes of Health, Neurim, Novartis, Otsuka, Oxford University Press, Pfizer, Physicians Postgraduate Press, Purdue, Roche, Sage, Shire, Sunovion, Supernus Pharmaceuticals, Transcept Pharmaceuticals, Validus, and WebMD. LG has received research support from the National Institute on Drug Abuse/National Institutes of Health, and Shire, and is on the advisory board for BioBehavioral Diagnostics. RJK is an employee of Rhodes Pharmaceuticals L.P. SW receives or has received research support and acted as a consultant and/or served on a speakers bureau for Attentiv, Eli Lilly, Forest Labs, Ironshore, Neos Therapeutics, NextWave Pharmaceuticals, the National Institutes of Health, Noven, NuTec, Otsuka, Pfizer, Purdue Pharma, Rho, Rhodes Pharmaceuticals L.P., Shiongi Pharma, Shire, Sunovion, Tris Pharma, and Vernalis.

\section{References}

1. Rhodes Pharmaceuticals. Biphentin ${ }^{\mathrm{TM}}$ Methylphenidate Hydrochloride Extended-Release Capsules for Treatment of Attention-Deficit Hyperactivity Disorder. Coventry (RI): Rhodes Pharmaceuticals; 2010.

2. Quinn D, Bode T, Reiz JL, Donnelly GA, Darke AC. Single-dose pharmacokinetics of multilayer-release methylphenidate and immediate-release methylphenidate in children with attention-deficit/hyperactivity disorder. J Clin Pharmacol. 2007;47(6):760-766.

3. Reiz JL, Donnelly GA, Michalko K. Comparative bioavailability of single-dose methylphenidate from a multilayer-release bead formulation and an osmotic system: a two-way crossover study in healthy young adults. Clin Ther. 2008;30(1):59-69.

4. Adjei A, Kupper RJ, Teuscher NS, et al. Steady-state bioavailability of methylphenidate extended-release (MPH-MLR) capsule vs immediaterelease methylphenidate tablets in healthy adult volunteers. Clin Drug Investig. 2014;34(11):795-805.

5. Adjei A, Teuscher NS, Kupper RJ, et al. Single-dose pharmacokinetics of methylphenidate extended-release multiple layer beads administered as intact capsule or sprinkles versus methylphenidate immediaterelease tablets $\left(\right.$ Ritalin $\left.^{\circledR}\right)$ in healthy adult volunteers. $J$ Child Adolesc Psychopharmacol. 2014;24(10):570-578.

6. Schachar R, Ickowicz A, Crosbie J, et al. Cognitive and behavioral effects of multilayer-release methylphenidate in the treatment of children with attention-deficit/hyperactivity disorder. J Child Adolesc Psychopharmacol. 2008;18(1):11-24. 
7. Weiss M, Hechtman L, Turgay A, et al. Once-daily multilayer-release methylphenidate in a double-blind, crossover comparison to immediaterelease methylphenidate in children with attention-deficit/hyperactivity disorder. J Child Adolesc Psychopharmacol. 2007;17(5):675-688.

8. Jain U, Hechtman L, Weiss M, et al. Efficacy of a novel biphasic controlled-release methylphenidate formula in adults with attentiondeficit/hyperactivity disorder: results of a double-blind, placebocontrolled crossover study. J Clin Psychiatry. 2007;68(2):268-277.

9. Stier EM, Davit BM, Chandaroy P, et al. Use of partial area under the curve metrics to assess bioequivalence of methylphenidate multiphasic modified release formulations. AAPS J. 2012;14(4):925-926.

10. Fourie Zirkelbach J, Jackson AJ, Wang Y, Schuirmann DJ. Use of partial AUC (PAUC) to evaluate bioequivalence - a case study with complex absorption: methylphenidate. Pharm Res. 2013;30(1):191-202.

11. US Food and Drug Administration. Draft guidance on methylphenidate hydrochloride. 2015. Available from: http://www.fda.gov/downloads/ Drugs/GuidanceComplianceRegulatoryInformation/Guidances/ UCM281454.pdf. Accessed January 5, 2015.

12. Shader RI, Harmatz JS, Oesterheld JR, Parmelee DX, Sallee FR, Greenblatt DJ. Population pharmacokinetics of methylphenidate in children with attention-deficit hyperactivity disorder. J Clin Pharmacol. 1999; 39(8):775-785.

13. Wigal SB, Gupta S, Greenhill L, et al. Pharmacokinetics of methylphenidate in preschoolers with attention-deficit/hyperactivity disorder. JChild Adolesc Psychopharmacol. 2007;17(2):153-164.

14. Wigal SB, Raja P, Shukla A. An update on lisdexamfetamine dimesylate for the treatment of attention deficit hyperactivity disorder. Expert Opin Pharmacother. 2013;14(1):137-145.
15. Kimko H, Gibiansky E, Gibiansky L, et al. Population pharmacodynamic modeling of various extended-release formulations of methylphenidate in children with attention deficit hyperactivity disorder via meta-analysis. J Pharmacokinet Pharmacodyn. 2012;39(2):161-176.

16. Center for Biologics Evaluation and Research, Center for Drug Evaluation and Research, Food and Drug Administration, US Department of Health and Human Services. Guidance for Industry: Population Pharmacokinetics. Silver Spring (MD): FDA; 1999. Available from: http://www.fda.gov/downloads/ScienceResearch/SpecialTopics/ WomensHealthResearch/UCM133184.pdf. Accessed January 5, 2015.

17. Rapport MD, Denney C. Titrating methylphenidate in children with attention-deficit/hyperactivity disorder: is body mass predictive of clinical response? J Am Acad Child Adolesc Psychiatry. 1997;36(4): 523-530.

18. Rodriguez W, Selen A, Avant D, et al. Improving pediatric dosing through pediatric initiatives: what we have learned. Pediatrics. 2008; 121(3): $530-539$.

19. DuPaul GJ, Power TJ, Anastopoulos AD, Reid R. ADHD Rating Scale-IV: Checklists, Norms, and Clinical Interpretation. New York: Guilford Press; 1998.

20. Wigal SB, Gupta S, Guinta D, Swanson JM. Reliability and validity of the SKAMP rating scale in a laboratory school setting. Psychopharmacol Bull. 1998;34(1):47-53.

\section{Publish your work in this journal}

Drug Design, Development and Therapy is an international, peerreviewed open-access journal that spans the spectrum of drug design and development through to clinical applications. Clinical outcomes, patient safety, and programs for the development and effective, safe, and sustained use of medicines are a feature of the journal, which

\section{Dovepress}

has also been accepted for indexing on PubMed Central. The manuscript management system is completely online and includes a very quick and fair peer-review system, which is all easy to use. Visit http://www.dovepress.com/testimonials.php to read real quotes from published authors.

Submit your manuscript here: http://www.dovepress.com/drug-design-development-and-therapy-journal 